\title{
Image-guided video-assisted thoracoscopic surgery with Artis Pheno for pulmonary nodule resection
}

\author{
Ya-Fu Cheng ${ }^{1 \#}$, Heng-Chung Chen ${ }^{1 \#}$, Pei-Cing Ke ${ }^{1}$, Wei-Heng Hung ${ }^{1}$, Ching-Yuan Cheng ${ }^{1}$, \\ Ching-Hsiung Lin ${ }^{2}$, Bing-Yen Wang ${ }^{1,3,4,5,6,7}$
}

${ }^{1}$ Division of Thoracic Surgery, Department of Surgery, ${ }^{2}$ Division of Chest Medicine, Department of Internal Medicine, Changhua Christian Hospital, Changhua; ${ }^{3}$ School of Medicine, College of Medicine, Kaohsiung Medical University, Kaohsiung, Kaohsiung; ${ }^{4}$ Institute of Genomics and Bioinformatics, ${ }^{5}$ Ph.D. Program in Translational Medicine, National Chung Hsing University, Taichung; ${ }^{6}$ School of Medicine, Chung Shan Medical University, Taichung; ${ }^{7}$ Center for General Education, Ming Dao University, Changhua

Contributions: (I) Conception and design: YF Cheng; (II) Administrative support: BY Wang; (III) Provision of study materials or patients: HC Chen; (IV) Collection and assembly of data: PC Ke, WH Hung; (V) Data analysis and interpretation: CY Cheng, CH Lin; (VI) Manuscript writing: All authors; (VII) Final approval of manuscript: All authors.

\#These authors contributed equally to this work.

Correspondence to: Bing-Yen Wang, MD, PhD. Division of Thoracic Surgery, Department of Surgery, Changhua Christian Hospital, No. 135 Nanxiao St., Changhua City, Changhua County 500, Changhua. Email: 156283@cch.org.tw.

Background: One challenging aspect of video-assisted thoracoscopic surgery (VATS) is finding the small pulmonary lung nodules for resection. Pre-operative localization of nodules is important for resection. Recently, image-guided VATS (iVATS) in a hybrid room has received attention. Our study aims to compare pros and cons between traditional CT room localization and iVATS localization with Artis Pheno.

Methods: This study was a retrospective analysis in our institute (Changhua Christian Hospital, Changhua). Patients with pulmonary nodules who received localization between January 2018 and December 2018 were included in the study. There were 126 patients included in the study. Among these, 63 patients received localization in a CT room and the other 63 patients received iVATS. We measured the time from localization to skin incision, success rate, complication rate, operation time, blood loss and length of hospital stay.

Results: Time from localization to skin incision was significantly shorter in the iVATS group than in the CT room group (23.57 vs. $372.11 \mathrm{~min}, \mathrm{P}<0.001$ ). The CT room group had a significantly higher complication rate than the iVATS group $(\mathrm{n}=49,77.8 \%$ vs. $\mathrm{n}=2,3.2 \%, \mathrm{P}<0.001)$. There were no significant differences in operation methods, operation time, blood loss and length of hospital stay.

Conclusions: iVATS provides shorter time from localization to skin incision and fewer complications than CT room localization.

Keywords: Image-guided; video-assisted thoracoscopic surgery (VATS); Artis Pheno; pulmonary nodule; localization

Submitted Aug 25, 2019. Accepted for publication Dec 10, 2019.

doi: $10.21037 /$ jtd.2020.02.12

View this article at: http://dx.doi.org/10.21037/jtd.2020.02.12

\section{Introduction}

Over the last decade, there have been two large trials indicating that low-dose computed tomography (CT) is an effective lung cancer screening tool for high-risk patients and its benefits outweigh its disadvantages $(1,2)$. There are more and more small nodules screened out. Further study of these small lesions is required. In recent years, videoassisted thoracoscopic surgery (VATS) has been proven to have better long-term outcomes and fewer postoperative complications than open thoracotomy for non-small cell 
lung cancer $(3,4)$. However, small nodules are challenging to remove due to finger palpation and instruments palpation being difficult during VATS. Therefore, removal of a small nodule requires precise localization of the lesion before the surgery.

Traditionally, these patients have to receive either blue dye or coil localization in the CT room. However, these patients will be at risk of pneumothorax and hemothorax after localization. The longer the wait is from localization to surgery, the greater the complication risk becomes. To overcome this difficulty, image-guided VATS (iVATS) in a hybrid room was first reported in 2013 (5). Several studies claim that iVATS provides a safe and efficient localization method compared to traditional localization $(6,7)$. The first uniportal iVATS was accomplished in 2016 (8). It was considered as an effective procedure.

However, it is difficulty to use the Artis Zeego robotic C-arm cone beam CT scanner (Siemens Healthcare $\mathrm{GmbH}$, Forchheim, Germany) due to the short radius of gyration (9). It requires profound experience to prevent the robotic $\mathrm{C}$-arm from colliding with a patient. The Artis Pheno robotic C-arm cone beam CT scanner (Siemens Healthcare GmbH, Forchheim, Germany) was introduced recently. It provides a longer radius of gyration to reduce the likelihood of collision and the number of scans.

Most studies reporting on iVATS involved traditional arms such as Artis Zeego or O-arm. There were no studies comparing pros and cons between traditional localization and iVATS localization with Artis Pheno. We therefore designed a retrospective review of our institution's experience of using iVATS with Artis Pheno to remove small lung nodules.

\section{Methods}

\section{Database}

This study was a retrospective analysis in our institute (Changhua Christian hospital, Changhua, Taiwan). All patients over 18 years of age undergoing removal of small lung nodules with pre-operative localization from January 2018 to December 2018 were included in the study. Tumors between 0.5 and $2.0 \mathrm{~cm}$ in size at peripheral were indicated for localization. Patients who did not receive localization, those who refused consent, and patients younger than 18 years of age were excluded. The patients were assigned to different groups by the participating time. Patients from January 2018 to June 2018 were assigned to the CT room group. Patients from July 2018 to December 2018 were assigned to the hybrid room group. This study was approved institutional review board in our institution (IRB190411) and written informed consent was obtained from all participants.

The following items were included in the study: age, gender, smoking status, tumor size, tumor location and localization method. We also included time from localization to skin incision, success rate, complication rate, operation time, blood loss and length of hospital stay as outcome measures.

\section{Study intervention}

Patients in the CT room group were admitted 1 day before surgery. The localization procedure was performed in the early morning before surgery by a board-certified and experienced radiologist. After localization, these patients were transferred to the general ward to wait for surgery. In the iVATS group, patients were informed to go to the operating room on the day of the operation. Localization was performed by either the attending surgeon or a radiologist, according to the surgeon's preference. We used robotic C-arm cone beam CT (Artis Pheno; Siemens Healthcare $\mathrm{GmbH}$, Forchheim, Germany) for the scanning. A total of 126 patients were included in the study. Among these, 63 patients received localization in a CT room and the other 63 patients received iVATS. All the patients in our study received uniportal VATS for the resection of pulmonary nodules.

\section{iVATS procedure}

All the patients in the iVATS group received scans in a hybrid room with the Artis Pheno robotic C-arm cone beam CT scanner (Siemens Healthcare GmbH, Forchheim, Germany) (Figure 1). After induction of general anesthesia, all patients were placed in true lateral decubitus position. A test scan was performed to ensure that the scanner would not collide with the patient. Another scan was performed with breath hold at end inspiration for surgical planning. We planned the location of needle incision and depth on the computer. A laser beam for incision location was created by syngo Needle Guidance of a syngo X-Workplace (Siemens Healthcare GmbH). We inserted an 18-gauge marker needle into the thorax with a laser-targeting cross projection guide, and the depth was measured before incision (Figure 2). Another scan was performed to confirm 


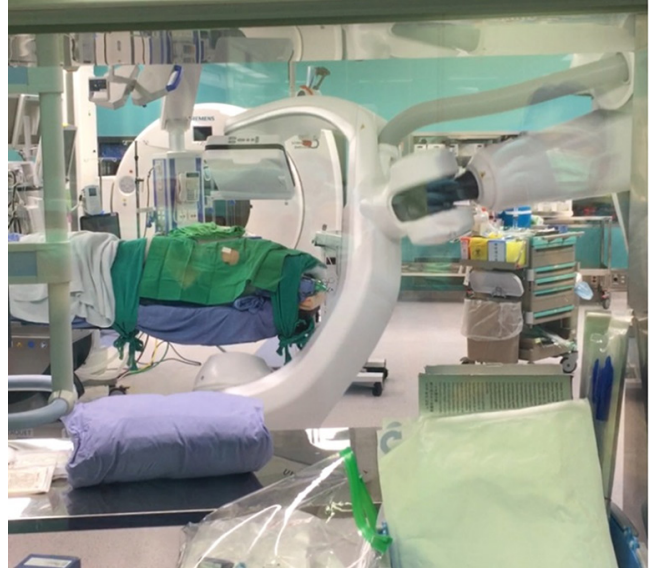

Figure 1 Hybrid room with robotic C-arm cone beam CT scanner (Artis Pheno; Siemens Healthcare GmbH, Forchheim, Germany).

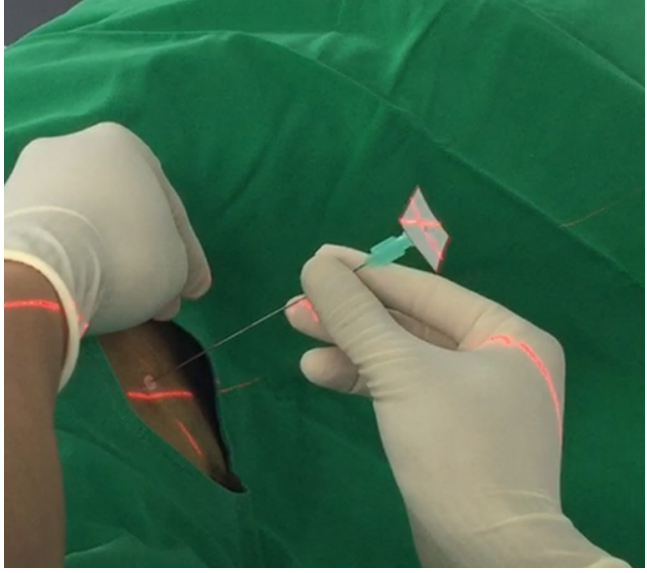

Figure 2 An 18-gauge marker needle is inserted into the thorax with a laser-targeting cross projection guide; the depth was measured before incision.
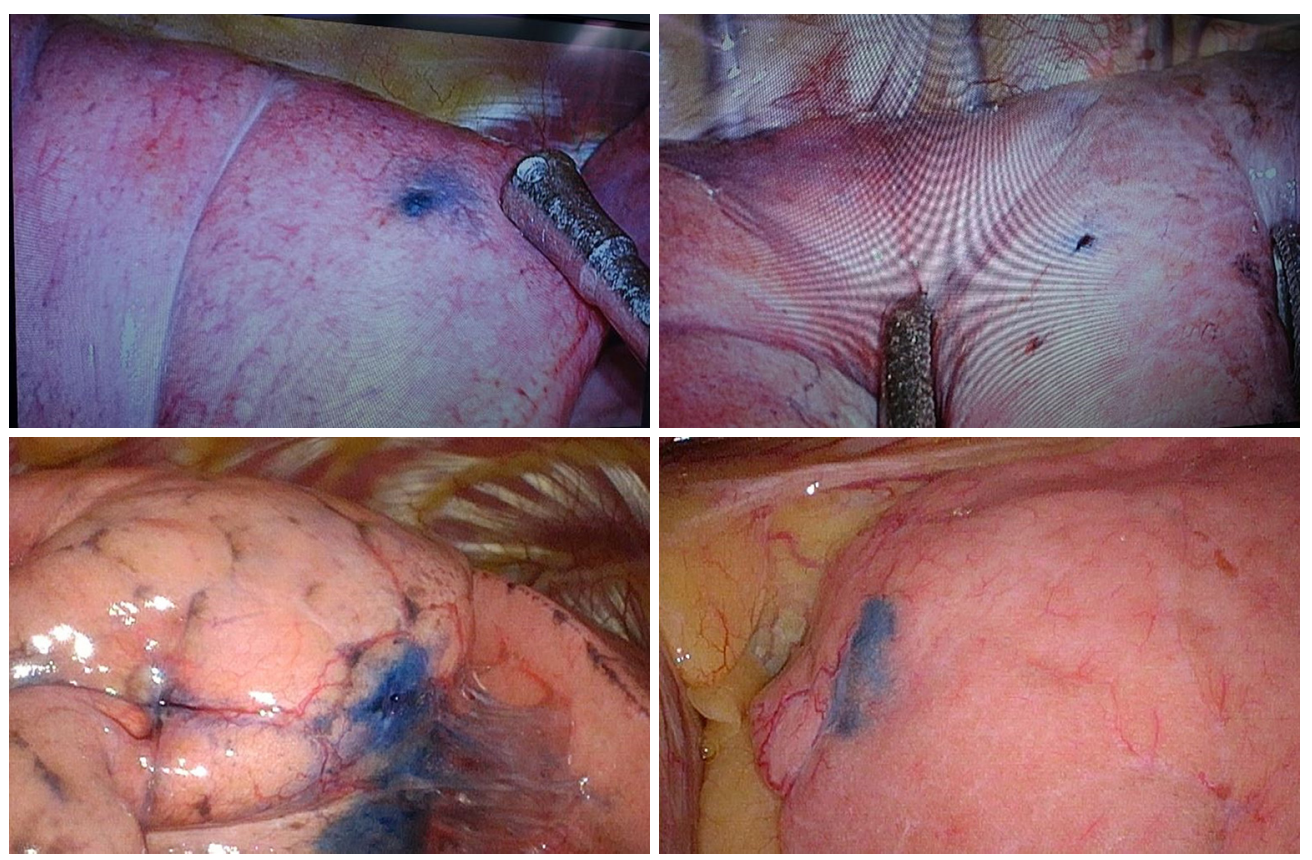

Figure 3 The lesion localization after $0.2 \mathrm{~mL}$ methyl blue dye injection.

the appropriate needle location. After the tip of the needle was at the appropriate place, we injected one of the following localization materials: diluted methyl blue dye $(0.2$ to $0.3 \mathrm{~mL})$, diluted indocyanine green (ICG) $(0.2$ to $0.3 \mathrm{~mL}$ ) or localization wire (Figure 3). The operation started after injection of the localization material and skin preparation.

\section{Statistical analysis}

We used SAS software (SAS System for Windows, version 9.2; SAS Institute, Cary, NC) to perform the statistical analysis for this study.

The outcome measures for our study were time from localization to skin incision, success rate, complication rate, operation time, blood loss and length of hospital 
Table 1 Basic data of patient characteristic

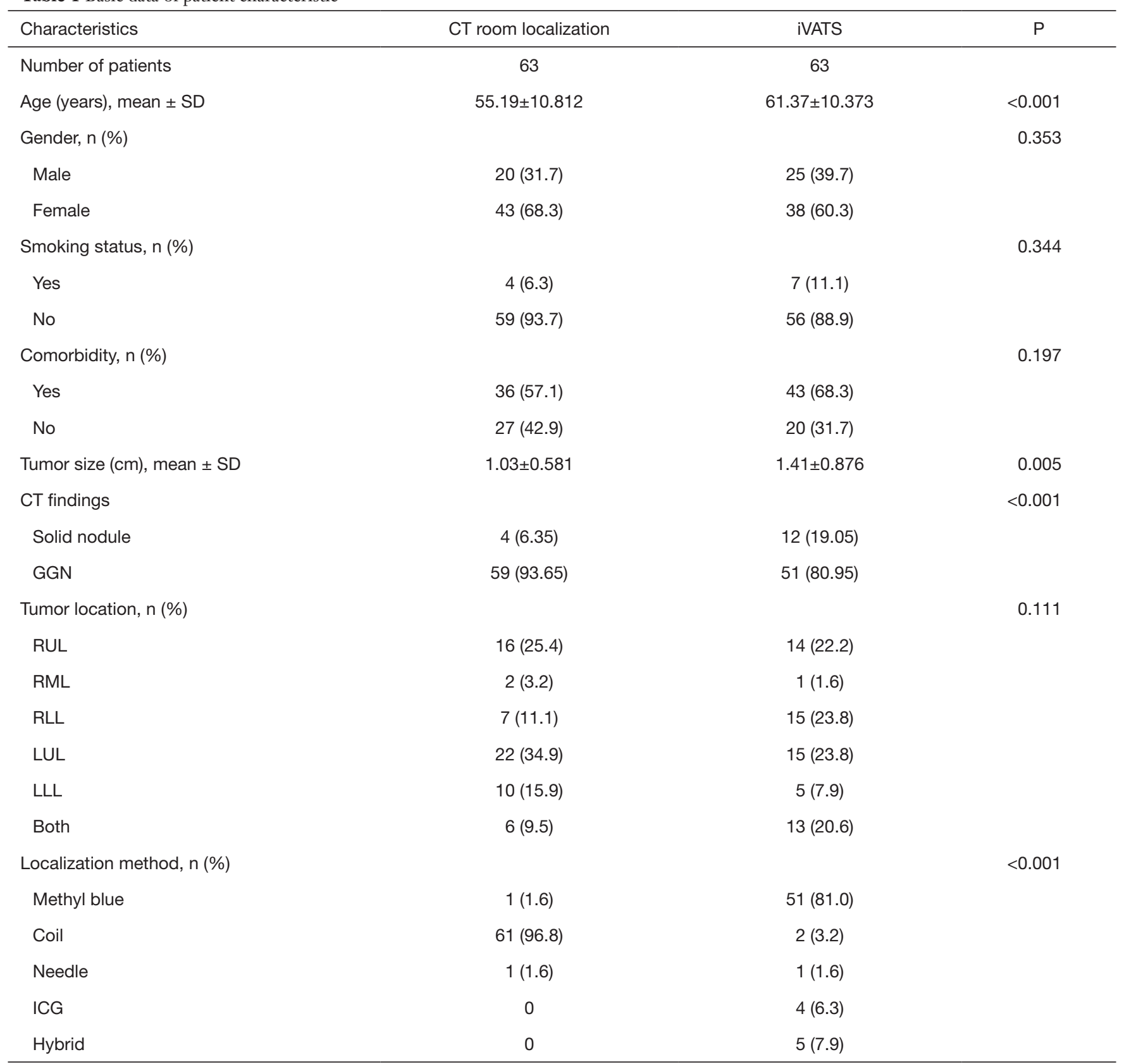

VATS, video-assisted thoracic surgery; GGN, ground-glass nodule; RUL, right upper lobe; RML, right middle lobe; RLL, right lower lobe; LUL, left upper lobe; LLL, left lower lobe.

stay. Time from localization to skin incision was defined as the time from the start of the pre-localization CT scan to skin incision in both groups. Surgery time was defined as the time from the first skin incision to the completion of surgical wound closure in both groups.

\section{Results}

Between January 2018 and December 2018, a total of 126 patients received localization for pulmonary nodule resection. 63 patients received localization in a CT room and the other 63 patients received iVATS (Table 1). The 
Table 2 Outcomes between CT room localization and iVATS

\begin{tabular}{|c|c|c|c|}
\hline Outcomes & CT room localization & iVATS & $\mathrm{P}$ \\
\hline Complication rate & 49/63 (77.8\%) & $2 / 63(3.2 \%)$ & $<0.001$ \\
\hline Pneumothorax & 30 & 2 & \\
\hline Hemorrhage & 4 & 0 & \\
\hline Successful rate & 63/63 (100\%) & $61 / 63(96.8 \%)$ & \\
\hline Operation methods, n (\%) & & & 0.064 \\
\hline Wedge resection & $41(65.1)$ & $47(74.6)$ & \\
\hline Segmentectomy & $19(30.2)$ & $8(12.7)$ & \\
\hline Wedge + segmentectomy & $1(1.6)$ & $3(4.8)$ & \\
\hline Operation time $(\mathrm{min})$, mean $\pm \mathrm{SD}$ & $119.46 \pm 57.771$ & $139.51 \pm 87.322$ & 0.073 \\
\hline Blood loss $(\mathrm{mL})$, mean \pm SD & $14.94 \pm 37.264$ & $45.65 \pm 180.637$ & 0.189 \\
\hline Length of hospital stay (day), mean \pm SD & $6.08 \pm 1.920$ & $6.08 \pm 2.720$ & 1.000 \\
\hline Cell type, n (\%) & & & 0.168 \\
\hline Adenocarcinoma & $45(71.4)$ & $33(52.4)$ & \\
\hline $\mathrm{SqCC}$ & $1(1.6)$ & $4(6.3)$ & \\
\hline Others & $2(3.2)$ & $3(4.8)$ & \\
\hline
\end{tabular}

ICG, indocyanine green; SqCC, squamous cell carcinoma.

iVATS group had older patients (55.19 vs. 61.37 years old, $\mathrm{P}<0.001$ ), and tumor size was larger in the iVATS group ( 1.03 vs. $1.41 \mathrm{~cm}, \mathrm{P}=0.005)$. Due to less experience of hybrid room localization, we chose larger tumor size in hybrid room localization at the beginning.

Over $90 \%$ of nodules are ground-glass nodules in CT room localization group. On the other hand, only about $80 \%$ of nodules are ground-glass nodules in the hybrid localization group $(\mathrm{P}<0.001)$. There were no significant differences regarding gender, smoking status, comorbidity, and tumor location.

The localization method was quite different in both groups. In the CT room group, most of the patients received coil insertion $(61 / 63,96.8 \%)$ because the color of methyl blue or ICG would fade after a long waiting time and thus make the lesion difficult to find. On the other hand, most of the patients in the iVATS group received methyl blue injection $(51 / 63,80.9 \%)$. The time from localization to skin incision was shorter in dye injection than coil. Because the patient was already in the OR, we needed to performed much sooner. The dyes for localization were more helpful to us. We mostly used methylene blue to lighten the financial burden on patients.

Table 2 summarized the outcomes of CT room localization and iVATS. Time from localization to skin incision in the iVATS group was significantly shorter than that of the CT room group (23.57 vs. $372.11 \mathrm{~min}, \mathrm{P}<0.001$ ). In addition, the complication rate was significantly higher in the CT room group compared to the iVATS group ( $\mathrm{n}=49$, $77.8 \%$ vs. $\mathrm{n}=2,3.2 \%, \mathrm{P}<0.001)$. Most patients in the CT room group suffered from pneumothorax and hemothorax by the last image of CT scan. Although there was no 
need for intervention in these 49 patients, some of them complained chest tightness and dyspnea. The satisfaction of patients decreased.

There were two failures of localization in the iVATS group due to inserting the needle in an inappropriate location. Pneumothorax was noted after removal of the needle, and it was difficult to localize again. However, we found a small bleeding hole on the surface of lung. This small hole could guide us to the place of the deviation area. We corrected the resection site by the guidance of this hole. For example, while the original needle puncture site was $2 \mathrm{~cm}$ below with $2 \mathrm{~cm}$ lateral to the nodule, we resected the lung higher and more medial. Larger resection area was also applied to make sure the safe margin due to failure of précised localization.

The two failures of localization could be finished with enough safe margin by the help of the small hole on the surface of lung. No conversion to open surgery or additional intervention was required.

The operation methods, operation time, blood loss, length of hospital stay and cell type were not significantly different among the two groups. There was no collision of the Artis Pheno robotic C-arm into a patient at true lateral decubitus position. Artis Pheno has a longer source-image distance (SID) $(130 \mathrm{~cm})$ compared to Artis Zeego $(120 \mathrm{~cm})$. The longer SID can accommodate heavier patients and true lateral position and increase patient entrance dose at interventional reference point.

The most common pathological cell type in both groups was adenocarcinoma (71.4\% in CT room group; $52.4 \%$ in iVATS group). After the frozen section confirmation of malignancy, we decided the operative methods based on both tumor size and the FEV1 of a pre-operative lung function test. All nodules confirmed with malignancy received both $\mathrm{N} 1$ and $\mathrm{N} 2$ lymph node sampling after confirmation.

\section{Comment}

Our study was the first study to compare the pros and cons between traditional CT room localization and iVATS localization with Artis Pheno. There were several studies reporting the iVATS experience with usage of Artis Zeego or O-arm (9-11). Most of the studies concluded that positioning the target involved difficulty and there was a substantial learning curve for preventing the $\mathrm{C}$-arm from colliding with patients. These difficulties did not exist in our experience with Artis Pheno. All the patients could be set at true lateral decubitus position without an ensuing collision due to the longer radius of gyration provided by Artis Pheno. Several previous studies with large case numbers reported that the time from localization to skin incision was more than 35 minutes $(8,12,13)$. Because there is little difficulty for the setting of patients' position with Artis Pheno, our study had a faster time from localization to skin incision $(23.57 \pm 16.541$ minutes) than previous studies. Moreover, Chao et al. reported that 38 consecutive iVATS procedures were needed to achieve stable procedural times and satisfactory success rates (14). With the help of Artis Pheno robotic C-arm, however, it would be easier for iVATS procedure.

The localization procedure during iVATS was conducted by either a thoracic surgeon or a radiology specialist. Thus, it requires little technique and can be completed by most doctors. In addition, Artis Pheno provides faster scanning time and image reconstruction time compared to Artis Zeego or O-arm. Due to Artis Pheno needing only 5 seconds for 360-degree scanning, it can also reduce the time from localization to skin incision.

We believe that this breakthrough of faster localization time can lead to a higher usage of iVATS in pulmonary resection worldwide. There were several methods reported for reducing collisions between robotic $\mathrm{C}$-arms and patients (9). The keys were to set patients in semi-prone or semi-supine position and to decrease the thickness of the table. However, all the patients in our study could be set at true lateral position due to the longer radius of gyration provided by Artis Pheno.

There are many benefits of iVATS surgery for patients with small pulmonary nodules. First, patients are under general anesthesia during localization, which reduces pain and anxiety. This can significantly improve the satisfaction of patients in regard to the whole hospital course. Second, the breath hold at end inspiration can be easily done with general anesthesia. This can lead to a lower failure rate of puncture and localization compared to local anesthesia during CT room localization. Third, the time from localization to skin incision is significantly short in iVATS. The corresponding low risk of pneumothorax and hemothorax provides safety to patients under localization. Furthermore, the whole procedure occurs in an operating room, which reduces the risk of transportation-related complications.

In our study, there are no significant differences between the CT room group and the iVATS group regarding operation methods, operation time, blood loss and length 
of hospital stay. The surgical procedure and post-operative care are similar. The most obvious difference is the different dying methods. In the CT room group, only coil localization is appropriate due to the color of methyl blue or ICG fading and thus making the lesion difficult to find. A previous study compared the pros and cons between micro coils and methylene blue injection for localization (15). Micro coils can have a success rate of up to $100 \%$ compared to $87 \%$ for methylene blue injection. However, micro coils induce a higher rate of pneumothorax and have a higher cost. We preferred dying localization because it is safe and it makes the location of the lesion easy to find, which saves lots of time. In the iVATS group, all of methyl blue, ICG and micro coil injection are appropriate localization methods. We tried these three methods, and we preferred methyl blue dying because it saves time and costs less for patients. Initially, we tried injections of $0.5 \mathrm{~mL}$ methyl blue according to suggestions from previous studies $(8,16)$. However, we found the dye spread out to the whole lobe, which caused difficulty in making the appropriate resection. In this condition, we tried to find the needle puncture hole at parietal pleura through the skin. With the calculation of depth from lesion to pleura, we speculated the appropriate resection area. To avoid this suboptimal scenario, we modified the methyl blue injection to $0.2 \mathrm{~mL}$ and subsequently rarely saw the dye spread out to the whole lobe (Figure 3).

This is the first study to report the experience of iVATS with Artis Pheno and compare it to the outcomes of CT room localization and iVATS with Artis Zeego. Nevertheless, there are some limitations of our study. First, this is a retrospective study. A prospective study is more convincing. Second, there exists some causes of bias such as age and tumor size. Tumor size was larger in the iVATS group. We need more time to collect more cases and to create a propensity match study. Third, the localization was performed by either the surgeon or radiology specialists. Each performer has a different level of experience. We need to eliminate the bias that comes from this. Lastly, tumors distance from the pleura was not recorded in our study. Though there were several limitations, our findings are important to show that there are several benefits in iVATS localization with Artis Pheno.

\section{Conclusions}

In conclusion, iVATS provides shorter time from localization to skin incision and fewer complications than
CT room localization. The Artis Pheno robotic C-arm cone beam CT scanner is designed to not easily collide with patients and provides easier pre-operative setting.

\section{Acknowledgments}

Funding: None.

\section{Footnote}

Conflicts of Interest: All authors have completed the ICMJE uniform disclosure form (available at http://dx.doi. org/10.21037/jtd.2020.02.12). The authors have no conflicts of interest to declare.

Ethical Statement: The authors are accountable for all aspects of the work in ensuring that questions related to the accuracy or integrity of any part of the work are appropriately investigated and resolved. This study was approved institutional review board in our institution (IRB190411) and written informed consent was obtained from all participants.

Open Access Statement: This is an Open Access article distributed in accordance with the Creative Commons Attribution-NonCommercial-NoDerivs 4.0 International License (CC BY-NC-ND 4.0), which permits the noncommercial replication and distribution of the article with the strict proviso that no changes or edits are made and the original work is properly cited (including links to both the formal publication through the relevant DOI and the license). See: https://creativecommons.org/licenses/by-nc-nd/4.0/.

\section{References}

1. Yousaf-Khan U, van der Aalst C, de Jong PA, et al. Final screening round of the NELSON lung cancer screening trial: the effect of a 2.5 -year screening interval. Thorax 2017;72:48-56.

2. National Lung Screening Trial Research T, Church TR, Black WC, et al. Results of initial low-dose computed tomographic screening for lung cancer. N Engl J Med 2013;368:1980-91.

3. Taioli E, Lee DS, Lesser M, et al. Long-term survival in video-assisted thoracoscopic lobectomy vs open lobectomy in lung-cancer patients: a meta-analysis. Eur J Cardiothorac Surg 2013;44:591-7.

4. Al-Ameri M, Bergman P, Franco-Cereceda A, et al. 
Video-assisted thoracoscopic versus open thoracotomy lobectomy: a Swedish nationwide cohort study. J Thorac Dis 2018;10:3499-506.

5. Narayanam S, Gerstle T, Amaral J, et al. Lung tattooing combined with immediate video-assisted thoracoscopic resection (IVATR) as a single procedure in a hybrid room: our institutional experience in a pediatric population. Pediatr Radiol 2013;43:1144-51.

6. Yang SM, Ko WC, Lin MW, et al. Image-guided thoracoscopic surgery with dye localization in a hybrid operating room. J Thorac Dis 2016;8:S681-9.

7. Hsieh MJ, Fang HY, Lin CC, et al. Single-stage localization and removal of small lung nodules through image-guided video-assisted thoracoscopic surgery. Eur J Cardiothorac Surg 2018;53:353-8.

8. Zhao ZR, Lau RW, Yu PS, et al. Image-guided localization of small lung nodules in video-assisted thoracic surgery. J Thorac Dis 2016;8:S731-7.

9. Hsieh MJ, Wen CT, Fang HY, et al. Learning curve of image-guided video-assisted thoracoscopic surgery for small pulmonary nodules: A prospective analysis of 30 initial patients. J Thorac Cardiovasc Surg 2018;155:182532.e1.

10. Yendamuri S, Demmy TL. Importance of mapping the

Cite this article as: Cheng YF, Chen HC, Ke PC, Hung WH, Cheng CY, Lin CH, Wang BY. Image-guided videoassisted thoracoscopic surgery with Artis Pheno for pulmonary nodule resection. J Thorac Dis 2020;12(4):1342-1349. doi: 10.21037/ jtd.2020.02.12 external environment in image-guided video-assisted thoracoscopic surgery. J Thorac Cardiovasc Surg 2018;155:1833.

11. Yu PSY, Man Chu C, Lau RWH, et al. Video-assisted thoracic surgery for tiny pulmonary nodules with realtime image guidance in the hybrid theatre: the initial experience. J Thorac Dis 2018;10:2933-9.

12. Chen PH, Hsu HH, Yang SM, et al. Preoperative Dye Localization for Thoracoscopic Lung Surgery: Hybrid Versus Computed Tomography Room. Ann Thorac Surg 2018;106:1661-7.

13. Chao YK, Wen CT, Fang HY, et al. A single-center experience of 100 image-guided video-assisted thoracoscopic surgery procedures. J Thorac Dis 2018;10:S1624-30.

14. Chao YK, Fang HY, Wen YW, et al. Intraoperative computed tomography-guided pulmonary tumour localization: a thoracic surgeon's learning curve. Eur J Cardiothorac Surg 2019;55:421-6.

15. Kidane B, Yasufuku K. Advances in Image-Guided Thoracic Surgery. Thorac Surg Clin 2016;26:129-38.

16. Fang HY, Chao YK, Hsieh MJ, et al. Image-guided videoassisted thoracoscopic surgery for small ground glass opacities: a case series. J Vis Surg 2017;3:142. 